\title{
Researching the Impact of Parameters of the Developed Routing Models on Network Performance
}

\author{
Mohammad Alhihi ${ }^{1}$, Hani Attar ${ }^{1}$, Mohammad Samour ${ }^{1}$, Artem Akulynichev ${ }^{2}$ \\ ${ }^{1}$ Philadelphia University, Amman, Jordan \\ ${ }^{2}$ National Aerospace University-Kharkov, Ukraine \\ Correspondence: Philadelphia University, Amman, Jordan.
}

Received: March 19, $2016 \quad$ Accepted: May 26, $2017 \quad$ Online Published: June 7, 2017

doi:10.11114/set.v4i1.2470 URL: https://doi.org/10.11114/set.v4i1.2470

\begin{abstract}
Nowadays, it is hard to imagine work without applying the principle of computer networks, and every day the requirement for high-quality network maintenance is increasing. In order to have a high-quality network; always optimize QoS through the optimization of routing protocols is required. In this paper, the scientific task of optimizing routing processes in hybrid telecommunication networks with guaranteed quality of service is solved by developing models and methods of adaptive routing. To develop methods, a system of Telecommunications network (TN) mathematical models was constructed at the levels of its morphological and functional descriptions. We used a weighted oriented graph as a structural model. Formalization of the main indicators of the network operation efficiency is carried out, which are the network performance (or its derivatives - relative and normalized performances) and indicators of the degree of use of network resources - buffer memory capacities of nodes and bandwidth of the transmission paths. In this paper, an experimental study of the developed models and routing methods was carried out in order to verify their adequacy, evaluate the effectiveness, and develop practical recommendations. The scheme of experiment, focusing on the investigation of processes occurring in the network while solving routing tasks (data gathering, RT processing, distribution, and implementation), is proposed.
\end{abstract}

Keywords: routing protocols, QoS, RT processing

\section{Introduction}

In communication networks, it is always important to understand the applications for the desired network, in term of power efficiency, transmission time, Quality of Service (QoS), data rate, ..., est. Each parameter mentioned above, requires a specific routing protocol to meet the required application needs. For example, The main parameters of the developed routing models, having a great impact on the efficiency of their practical implementation, are a value of recalculation range of the route variables $\Delta t$ and a number of the recalculation ranges $a$, involved in the formation of target function, which eventually define the value of forecast period $T=a \Delta t$.

The influence of parameter $\Delta t$ on TN functioning efficiency is twofold. On the one hand, $\Delta t$ should be as small as possible to provide the high flexibility of response of the developed routing models to network condition: load of queue buffers on the nods, network bandwidth, and incoming traffic. On the other hand, reduction of $\Delta t$ involves a significant increase in overhead amount that is network status and control information. Routing implementation in accordance with the developed models may become impractical at exceeding of certain limit of overhead amount, therefore the minimum value of $\Delta t$ should be limited to certain value $\Delta t_{\mathrm{min}}$. For practical reasons, a prerequisite for implementation of the developed routing models is a fulfillment of condition $\Delta t \geq \Delta t c o n$, where $\Delta t c o n$ is the control cycle duration imposed by Anastasi, Giuseppe (2009) .Thus, the lower limit of recalculation range of route variables is defined as a highest value among $\Delta t_{\min }$ and $\Delta t_{\text {Con }}$. 
At the same time, increasing in $\Delta t$ leads to a routing strategy change and a shift from a dynamic routing to quasi-static or, in the limit $\Delta t \rightarrow \infty$, to static. Depending on the chosen strategy, the upper limit of $\Delta t$ can be defined: $\Delta t_{\max }$. Such wise, the chosen routing strategy and the related quantities of overhead streams define the upper and the lower limits while choosing the value of the recalculation range of route variables $\Delta t: \Delta t_{\min } \leq \Delta t \leq \Delta t_{\max }$. As operating practice of IP-networks El-Hihi, Mohamed(2016), Alhihi, Mohammad(2017) Network Coding, Attar, Hani and Mohammad Alhihi 2014 and H. Attar, L. Stankovic and V. Stankovic(2011)the minimum value of recalculation period is a few tens of seconds, the maximum value, as will be shown later, depends on the nature of load incoming to maintenance to a larger extent than on network features.

Parameter $a$ defines the degree of forecast feature implementation of the developed routing models. At the same time, by analogy with value $\Delta t$, the upper and the lower limits of the number of recalculation ranges can be defined: $a_{\min } \leq a \leq a_{\max }$, besides $a_{\min }=1$ which corresponds to the absence of network status forecast. There are several reasons for implementation of $a>1$ : first, there is need to avoid occurrence of loops in calculated routes, second, processes of backup and restoring of network resources are scheduled. From a computational point of view, increasing in $a$ leads to a proportional increase in dimension of the optimization problem Raju, Jyoti, and Jose Joaquin Garcia-Luna-Aceves(1999).and Liebig, Thomas(2017), in practical terms - it leads to a necessity of forecasting with the given accuracy subscribers' data load on the network nodes. Thereby, parameter $a$ is restricted by the value of $a_{\max }$ which is corresponding with the most favorable conditions for implementation of the considered routing method. Within this work no analytical justification for choosing parameter $a$ was made. Given the complexity of analytical justification for choosing parameters $\Delta t$ and $a$, it becomes necessary to conduct experimental studies of an effect of these parameters onto network performance in general, the results of which are advisable to clarify in practical realization.

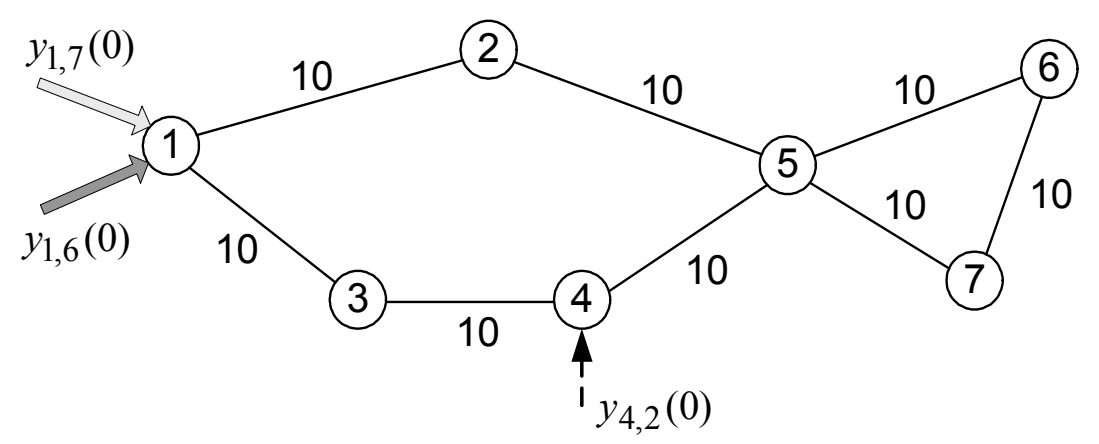

Figure 1. Example for sub-optimal distribution network protocol

As mentioned and illustrated with the example Fig. 1 above, the most benefit from implementation of the developed routing models is observed when using it in irregular network structures. Considering these structures became most widespread in practice, let's choose the networks shown in Fig. 2 for further network simulation. Simulation results confirm the results obtained with $N=5$ and $N=7$ for networks with a large number of nodes and testify the high efficiency of the developed models as compared with the shortest path models. Numerical values of capacities of the transmission paths Fig. 2 are caused by TN construction practice and equal 200 Mbps.

To explore an impact of the model parameters on routing quality we'll build a simulation considering the network example Fig. 2a. The total simulation time is $T_{\mathrm{M}}=100 \mathrm{~s}$. Four main options of the routing model implementation, differing in the value of the recalculation range of route variables $\Delta t$ and its number $a$ Table 1, were considered in the simulation. Option 1 corresponds to the most frequent update of routing tables, and in case of option 4 the route variables hadn't changed throughout the simulation time. 


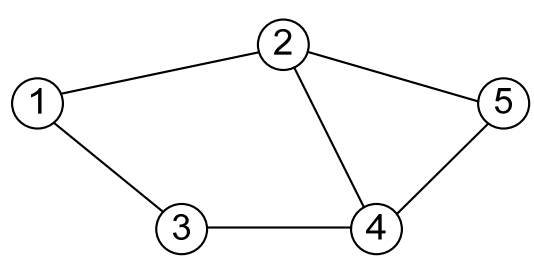

(a)

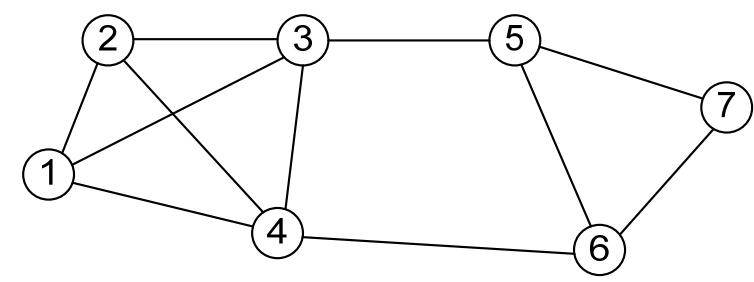

(b)

Figure 2. Network structures used in the simulation

Table 1. Model parameters

\begin{tabular}{ccc}
\hline Option & $\Delta t, \mathrm{~s}$ & $a$ \\
\hline 1 & 10 & 10 \\
2 & 20 & 5 \\
3 & 50 & 2 \\
4 & 100 & 1 \\
\hline
\end{tabular}

Simulation consists of $v=a$ steps for all the options, the result of calculations for each step, as mentioned in Akkaya, Kemal, and Mohamed Younis(2005),is the set of $v=a$ vectors of the route variables $U(k), k=\overline{0, a-1}$, only $U(0)$ of which will be implemented. Let's assume the simulation result is precisely the vectors that will be implemented. Thus, the simulation result for each of the options is the set of $v=a$ route vectors defining the contents of appropriate routing tables and therefore the resource allocation over time $T_{\mathrm{M}}$. The elements of the route vectors were gained by solving the minimizing-oriented optimization problem $W$. The objective-functional view of $W$ for each of the options will match its parameter $a$ value listed in Table 1.

$$
W=\sum_{k=0}^{a-1}\left[X^{T}(k) Q_{X} X(k)+U^{T}(k) Q_{U} U(k)\right] \rightarrow \min
$$

Thus obtained route variables allow us to estimate the network performance (Fig. 2) by means of Elman, Jeffrey L(1990). and Jang(1993), the usage rate of network channel resources in total Fig. 3 by means of Eq. 1. Here, to compare the simulation results, obtained for the different options, let's take $\Delta t_{1}=10 \mathrm{~s}$ as a time interval unit. Fig.2 shows us the dependences of the brought data amounts within the simulated network during the time interval unit on its number throughout the time of simulation $k=\overline{1,10}$ (for convenience, the numbering of time intervals was started not with 0 but 1). Here curves 1-4 correspond with $P(k) \Delta t_{1}$ value of the considered options Table 1. For comparison, Fig.4 shows the dynamics of changes in the amounts of load coming into the network during the corresponding time interval unit $Y_{\mathrm{BX} \Sigma}(k)$ (curve 5).

Fig.3 shows the dynamics of usage rate of the network bandwidth $K u(k)$ depending on the number of the time interval unit $\Delta t_{1}$. Usage rate of channel resources changes in accordance with the specified recalculation interval of routing tables ( $\Delta t$ in Table 1) and the dynamics of load amounts coming into the network. As the obtained results show, the 
mean of $K u$ throughout the simulation time for all the cases is approximately the same, its highest dynamics occurs in case of the lowest $\Delta t$ (option 1).

Comparison of the dynamics of change of $Y_{\mathrm{BX} \Sigma}(k)$ and $K u(k)$ in point of the option 1 of the routing model indicates that allocated network resources are just enough to service the traffic, being received during $\Delta t$, without losses. In case of $\Delta t$ increasing (dependences 2-4), the changes of $K u$ no longer correspond with the load changes. As a result, a delay of the received traffic occurs that in a real network corresponds with the queue growth and therefore the value of losses and overloads increasing, which ultimately reduces the quality of service.

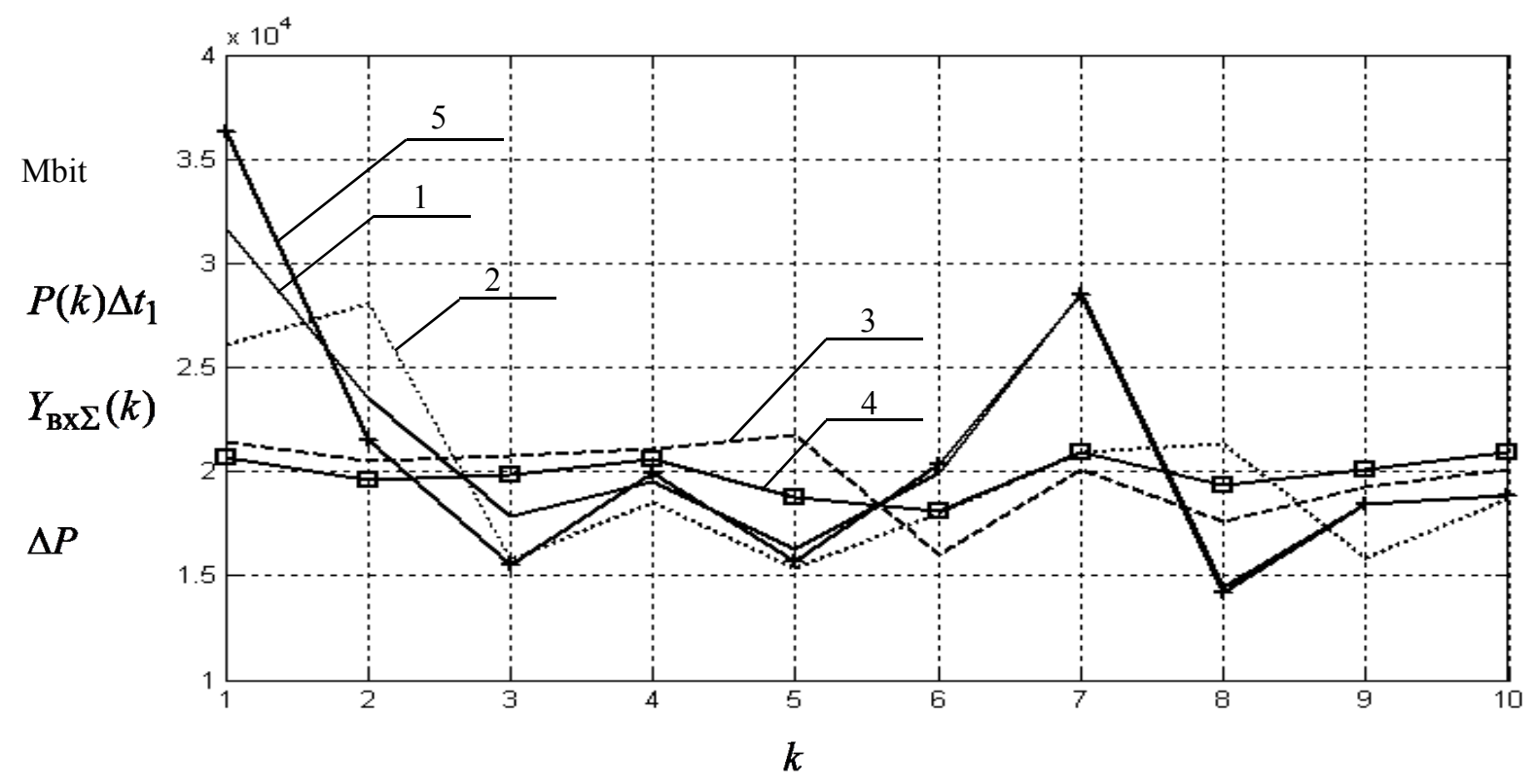

Figure 3. The dynamics of change of the incoming load $Y_{\mathrm{Bx} \Sigma}(k)$ and the network performance $P(k) \Delta t_{1}$

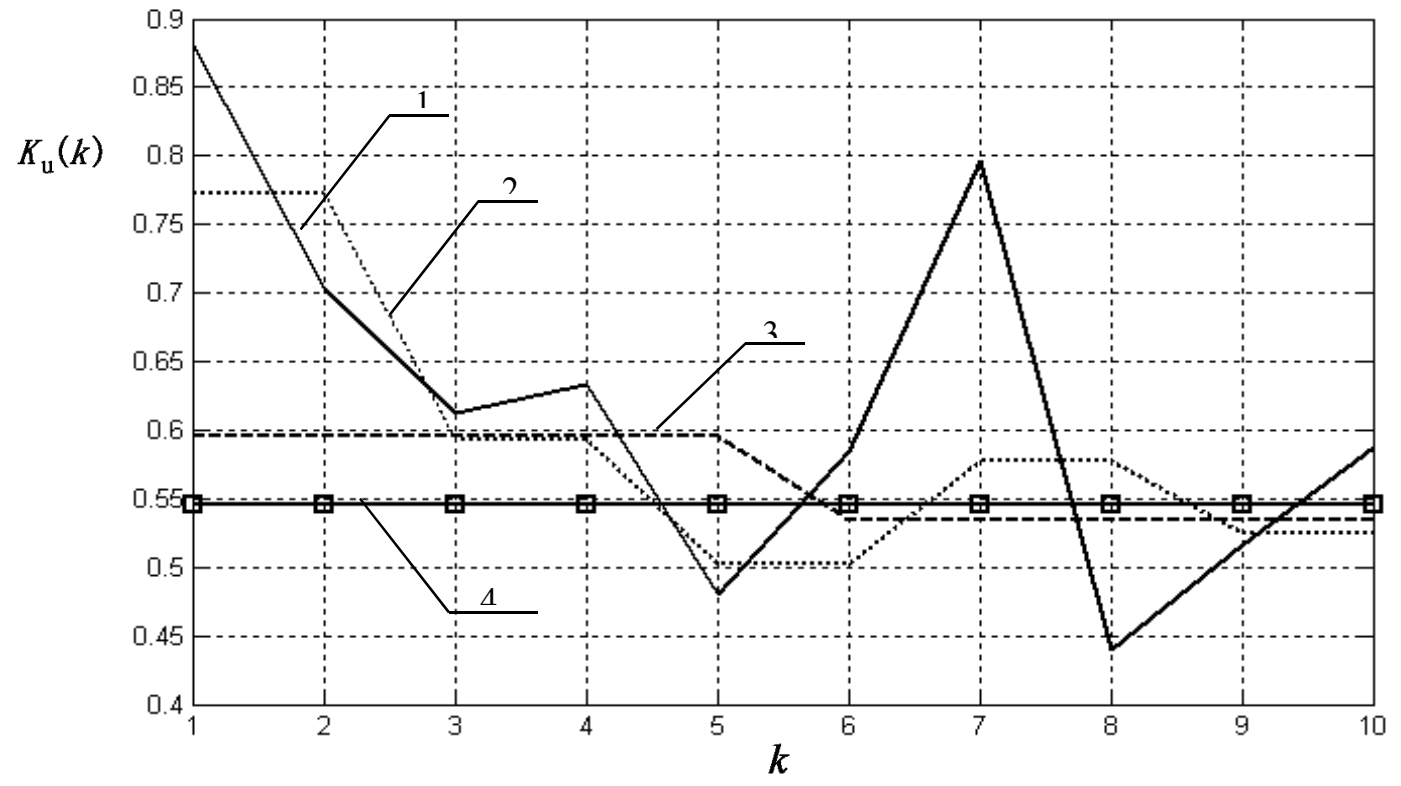

Figure 4. The dynamics of change of the usage rate of network channel resources $K u$ 


$$
\begin{gathered}
\Delta P=\frac{\sum_{h=1}^{v_{1}} Y_{\mathrm{BX} \Sigma}(h)-\Delta t_{1} \sum_{h=1}^{v_{1}} P(h)}{\sum_{h=1}^{v_{1}} Y_{\mathrm{BX} \Sigma}(h)}, \\
v_{1}=\frac{T_{\mathrm{M}}}{\Delta t_{1}},
\end{gathered}
$$

As a generalization of the obtained results, (Fig.4) shows a diagram estimating the lost productivity amount as a relative amount of data undeceived by user throughout $T_{\mathrm{M}}$ for the options 1-4. Fig. 5 shows that the highest network performance is reached in case of option 1 because of the smallest recalculation interval of routing tables in conditions of the incoming load high dynamics.

Lost productivity value $\Delta P$ is greatly affected by a degree of unevenness of the incoming load $y_{\mathrm{BX}} i, j(k)$ due to the change of $\Delta t$. As an estimate of load unevenness we'll use a standard deviation $\sigma_{y i, j}(k)$ which is a distribution-law parameter in accordance with which the simulation of random load process was executed. Fig.5 shows the diagrams of the lost productivity value $\Delta P$ of a full-mesh network having $N=5$ nodes depending on the $\Delta t$ and $\sigma_{y}$ values under the assumption that $M\left[y_{\mathrm{Bx} i, j}(k)\right]=y_{\mathrm{cp}}, D\left[y_{\mathrm{BX}} i, j(k)\right]=\sigma_{y}^{2}$. Hence it is clear that, considering $\sigma_{y}$ not bigger than $20 \%$ of $y_{c p}$ mean value, $\Delta P$ is no more than $2 \% . \Delta P$ increases to $5 \%$ with $0,65 y_{c p}$ and is $8.5 \%$ with $1,2 y_{c p}$.

Analysis of the simulation results allows to make the following conclusion Yevseeva O.Yu(2002). the choice of the recalculation period of routing tables $\Delta t$ is defined by change intensity of the incoming network load and should be equal to the time during which the incoming load can be approximately regarded as constant. Particular value should be specified for the real network conditions considering not only incoming load dynamics but also the amount of generating overhead. If for transmission of overhead the separate signaling network is used, $\sigma_{y}$ and the control cycle duration $\Delta t c o n$ will be the determining factor in choosing $\Delta t$. When using information channels for transmitting overhead packets, a network load created by them, amount of which depends on size of overhead packets, implemented overhead change protocol and which increases with the decreasing of $\Delta t$, should be considered. In this case $\Delta t$ is chosen as a compromise between the losses $\Delta P$, caused by $\sigma_{y}$, and the overhead amounts. As the results of simulation of the QoS-routing method, built on the basis of the OSPF protocol Apostolopoulos G, Guerin R, Kamat S(1999), Yang, Lei(2016),Domżał Jerzy et al(2015) and Alhihi, Mohammad(2017) Practical Routing Protocol Models show, the band needed for overhead transmission is $30 \mathrm{kbps}$ (the maximum is $174 \mathrm{kbps}$ ) on the average in case of the channel capacity of $45 \mathrm{Mbps}$ and the eight-node structure. This allows to conclude that the determining factor when choosing $\Delta t$ is $\sigma_{y}$ and $\Delta t c o n$. 


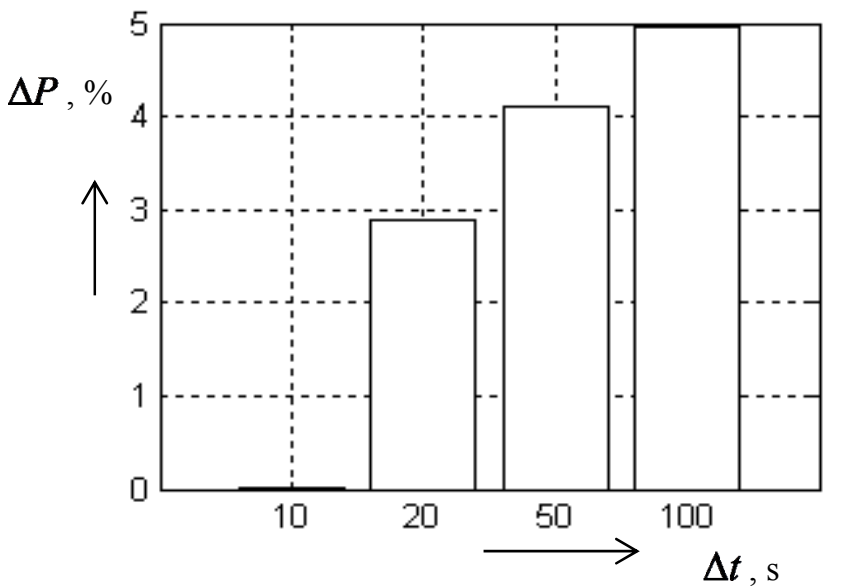

Figure 5. Diagram of the lost productivity value $\Delta P$ of the options $1-4$ Table $2\left(\sigma_{v}=0,65 y_{c p}\right)$

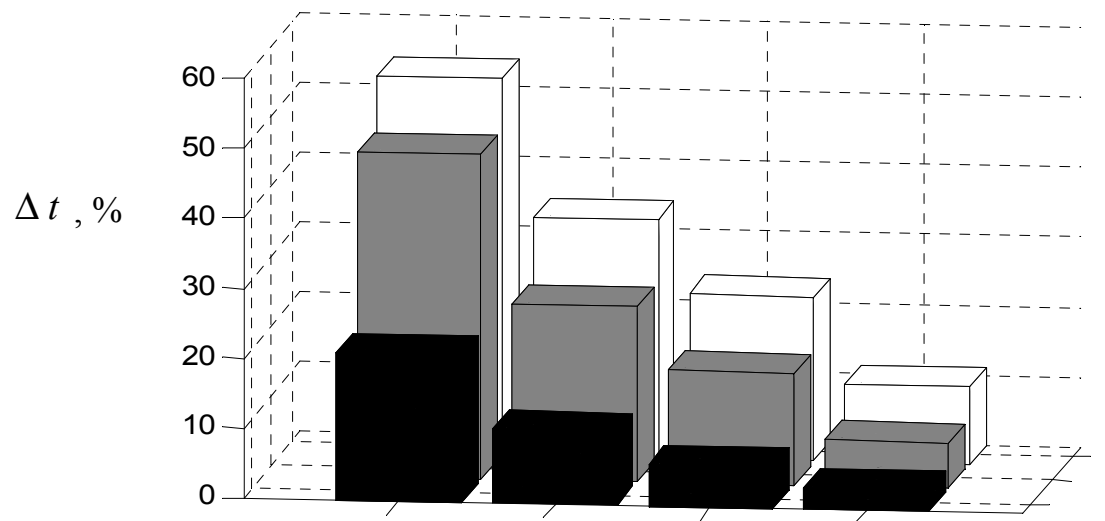

Figure 6. Diagram of the lost productivity value $\Delta P$ of a network having $N=\stackrel{\Delta t, \mathrm{c}}{y_{\mathrm{cp}}}$ $\sigma_{y}$ values.

To evaluate an impact of the parameter $a$, which is the number of the recalculation intervals of routing tables, on the solutions for routing tasks, let's build a simulation considering the networks shown in (Fig.1) Let us consider the dependence of network performance on the incoming traffic amounts and the $a$ value. It is assumed here that $M\left[y_{\mathrm{Bx} i, j}(k)\right]=y_{\mathrm{cp}}, D\left[y_{\mathrm{BX} i, j}(k)\right]=\sigma_{y}^{2}, \sigma_{y}=0,6 y_{c p}$, and $\Delta t=10 \mathrm{~s}$.

As evinced by the results of simulation (Figs. 6, 7), implementation of the forecasting properties of the routing models improves TCN productivity by $\Delta P_{a}=\frac{P_{a}-P_{1}}{P_{1}} 100 \%$, where $P_{a}$ and $P_{1}$ are the network performance in case of implementation of the routing method with and without forecast $\left(a=a_{\min }=1\right)$ respectively. An average performance increase through implementation of the forecasting properties is 14-18\%. With increasing in $a$ Fig. 7 the rate of productivity growth slows down, and with $a>5$ the increase in productivity through implementation of the forecasting properties is insignificant. Since the increase in $a$ is associated with the increase in transmitted overhead amounts, we can conclude that it is advisable to limit forecast interval to a value close to $a=5$. 
To analyze the forecast properties in overload mode let's consider the behavior of the model at an income load overcoming the network bandwidth H. Attar, L. Stankovic, M. Alhihi and A. Ameen(2014), and Attar, H. (2017) Data Combination .Let's consider two options of the adaptive routing model on an example of the network shown in Fig.1a with parameters $a=5$ and $a=1$, i.e. a model that has the forecast property (option 1) and does not have that (option 2).

The total simulation time is $T_{\mathrm{M}}=50 \mathrm{~s}$, the interval is $\Delta t=10 \mathrm{~s}$. The obtained dependences Figs. 7 and 8 indicate that during the first time interval the routing model without forecasting (curve 2) exceeds the forecast model (curve 1) by performance but stays inferior in the remaining time. This is explained by the different strategies implemented by the options under consideration. Model without forecasting provides a solution to the routing task at each time interval through the use of communication directions mainly for delivering flows on direct routes. In the interval $k=1$ this gives a certain gain, but then, with $k$ growth, in view of the "ignoring" of queues, for unloading of which there are no direct routes, an overload of individual directions and then of the network as a whole occurs. Model with forecasting at the first steps of the work $(k=1)$ already organizes not only direct but also bypass routes, i.e. a proportion of the delivered traffic, for which there are direct communication ways, decreases. Thereby, the total amount of delivered information within $k=1$ becomes smaller, but at the subsequent time intervals $k=4 \ldots 5$, when the network with model 2 is already close to overload mode or entered it, model 1 ensures the delivery of user information without losses. For example, Figs. 7 and 8, show the change in the network load, estimated by the total value of all network queues
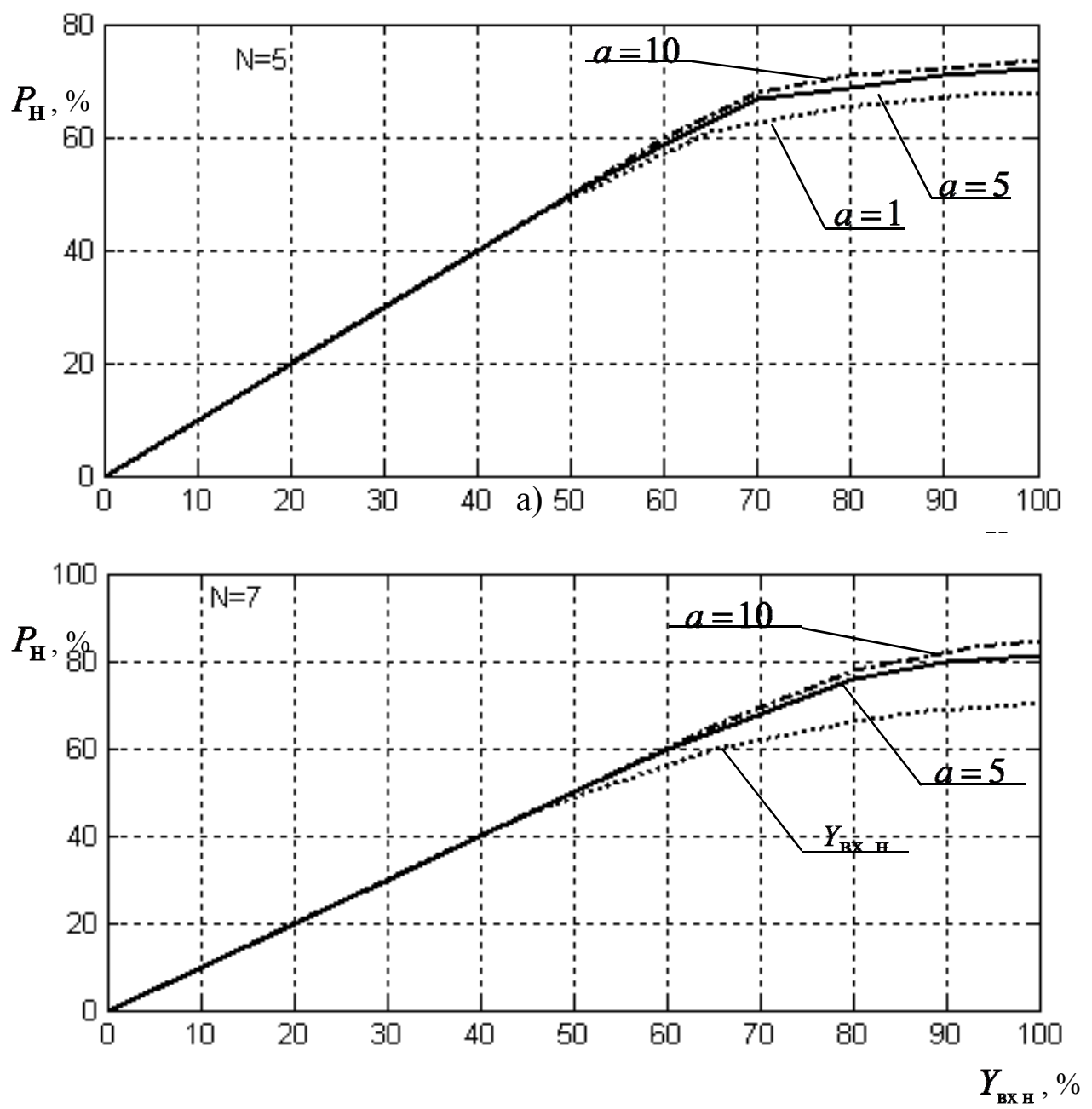

Figure 7. Dependence of the normalized network performance on the network load for various $a$ 


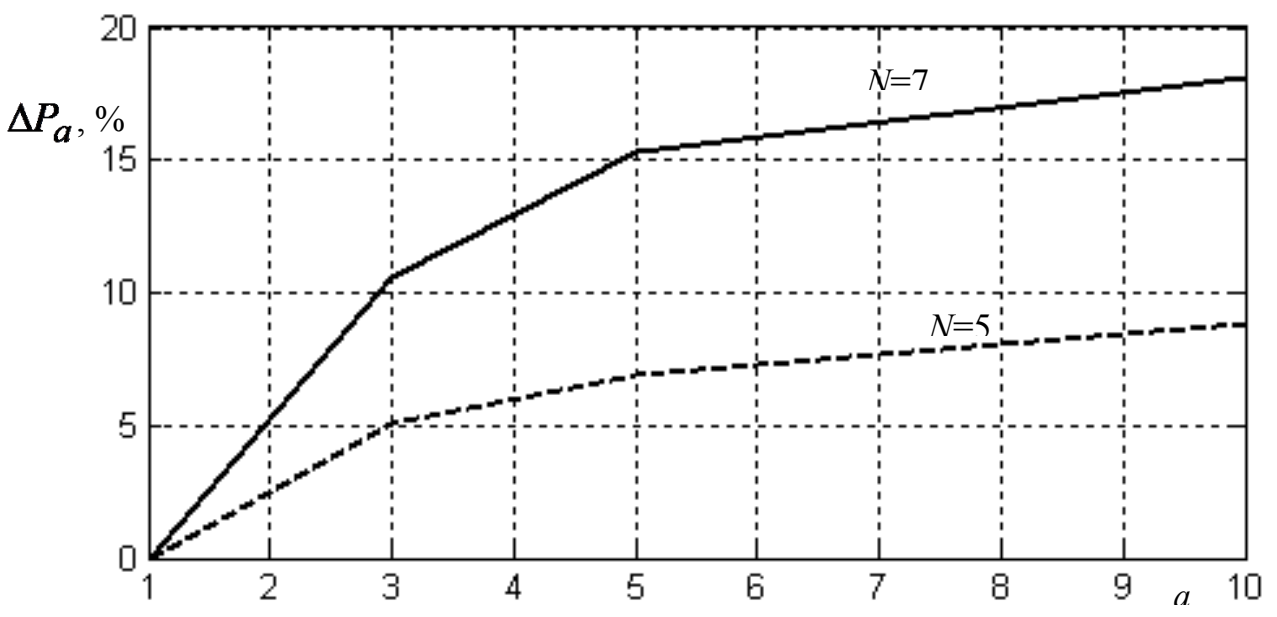

Figure 8. Dependence of increase in the network performance on the parameter $a \sum_{\substack { i=1 \\ \begin{subarray}{c}{j=1, j \neq i{ i = 1 \\ \begin{subarray} { c } { j = 1 , \\ j \neq i } }\end{subarray}}^{N} x_{i, j}(k)$, which indicates

that routing without forecasting allows entering the overload mode somewhat earlier Yevseeva O.Yu(2002).

\section{Conclusions}

The influence of individual model parameters on the efficiency of its implementation was investigated, and it was established that the greatest influence is exerted by the recalculation period of route variables $\Delta t$ and the forecasting interval $a$, the choice of which should be coordinated. Formalization of the main indicators of the network operation efficiency is carried out, which are the network performance (or its derivatives - relative and normalized performances) and indicators of the degree of use of network resources - buffer memory capacities of nodes and bandwidth of the transmission paths. An experimental study of the developed models and routing methods was carried out in order to verify their adequacy, evaluate the effectiveness, and develop practical recommendations. The scheme of experiment, focusing on the investigation of processes occurring in the network while solving routing tasks (data gathering, RT processing, distribution, and implementation), is proposed. The influence of individual model parameters on the efficiency of its implementation was investigated, and it was established that the greatest influence is exerted by the recalculation period of route variables $\Delta t$ and the forecasting interval $a$, the choice of which should be coordinated. As shown by the simulation results Fig. 7, it is advisable to limit the forecasting period to a value of five.

\section{References}

Akkaya, K., \& Mohamedm, Y. (2005). A survey on routing protocols for wireless sensor networks. Ad. Hoc. Networks, 3(3), 325-349. https://doi.org/10.1016/j.adhoc.2003.09.010

Alhihi, M. (2017). Network Coding for Wireless Sensor Network Cluster over Rayleigh Fading Channel: Finite State Markov Chain. International Journal of Communications, Network and System Sciences 10(1), 1. https://doi.org/10.4236/ijens.2017.101001

Alhihi, M. (2017). Practical Routing Protocol Models to Improve Network Performance and Adequacy. Journal of Computer and Communications, 5(6), 114. https://doi.org/10.4236/jcc.2017.56007

Anastasi, G. et al. (2009). Energy conservation in wireless sensor networks: A survey. Ad. Hoc. Networks, 7(3), 537-568. https://doi.org/10.1016/j.adhoc.2008.06.003

Apostolopoulos, G., Guerin, R., \& Kamat, S. (1999). Implementation and Performance Measurements of QoS Routing Extentions of OSPF // Proc. IEEE INFOCOM. - New York (USA), 680-688.

Attar, H. (2017). Data Combination over Physical Layer Using Network Coding with PUM Turbo Codes. Journal of Computer and Communications, 5, 32-44. https://doi.org/10.4236/jcc.2017.56002

Attar, H. et al. (2014). Deterministic network coding over Long Term Evaluation Advance communication system. Digital Information and Communication Technology and it's Applications (DICTAP), Fourth International 
Conference on. IEEE, 2014. https://doi.org/10.1109/dictap.2014.6821657

Attar, H., Stankovic, L., \& Stankovic, V. (2011). Cooperative network-coding system for wireless sensor networks, IET Communications, 6(3), 344-352. doi: 10.1049/iet-com..0143

Attar, H., Stankovic, L., Alhihi, M., \& Ameen, A. (2014). Deterministic network coding over Long Term Evaluation Advance communication system, 2014 Fourth International Conference on Digital Information and Communication Technology and its Applications (DICTAP), Bangkok, 56-61. https://doi.org/10.1109/DICTAP.2014.6821657

Domżał, J., et al (2015). A survey on methods to provide multipath transmission in wired packet networks. Computer Networks, 77, 18-41. https://doi.org/10.1016/j.comnet.2014.12.001

El-Hihi, M. et al. (2016). Network Coding Cooperation Performance Analysis in Wireless Network over a Lossy Channel, M Users and a Destination Scenario, Communications and Network, 8(4), 257. https://doi.org/10.4236/cn.2016.84023

Elman, J. L. (1990). Finding structure in time. Cognitive Science, 14(2), 179-211. https://doi.org/10.1207/s15516709 $\operatorname{cog} 1402 \_1$

Jang, J. (1993). ANFIS: adaptive-network-based fuzzy inference system. IEEE transactions on systems, man, and cybernetics, 23(3), 665-685. https://doi.org/10.1109/21.256541

Liebig, T. et al. (2017). Dynamic route planning with real-time traffic predictions. Information Systems, 64, 258-265. https://doi.org/10.1016/j.is.2016.01.007

Raju, J., \& Garcia-Luna-Aceves, J. J. (1999). A new approach to on-demand loop-free multipath routing. Computer Communications and Networks, 1999. Proceedings. Eight International Conference on. IEEE. https://doi.org/10.1109/iccen.1999.805568

Yang, L. (2016). The Open Automation and Control Systems Journal. Open Automation and Control Systems Journal, 8 , 94-102. https://doi.org/10.2174/1874444301608010094

Yevseeva, O. Y. (2002). Experimental study of the dynamic model of adaptive routing in datagram telecommunication networks. // Radiotekhnika: Vseukr. Interd. Scientific-techn. Sat, 133, 20-26.

\section{Copyrights}

Copyright for this article is retained by the author(s), with first publication rights granted to the journal.

This is an open-access article distributed under the terms and conditions of the Creative Commons Attribution license which permits unrestricted use, distribution, and reproduction in any medium, provided the original work is properly cited. 